\title{
Old and New TB Drugs: Mechanisms of Action and Resistance
}

\author{
Anastasia S. Kolyva and Petros C. Karakousis \\ Johns Hopkins University Center for Tuberculosis Research \\ Baltimore, M.D., \\ USA
}

\section{Introduction}

Historically, tuberculosis (TB) has been associated with significant morbidity and mortality, and still remains a major global health problem. It is estimated that 2 billion people are latently infected with Mycobacterium tuberculosis, resulting in approximately 3 million deaths worldwide per year. Among the unique features of this organism is its ability to establish persistent infection, requiring prolonged antibiotic treatment in order to achieve clinical cure. The basic goals of anti-tuberculosis therapy include rapid killing of actively multiplying bacilli, prevention of acquired drug resistance, and sterilization of infected host tissues to prevent clinical relapse. Official guidelines recommend a minimum of 6 months of combination antibiotic therapy in order to achieve these goals. Clinical isolates in geographic areas with a high prevalence of drug resistance should be tested routinely for susceptibility to first-line anti-tuberculosis agents if resources permit, in order to optimally guide therapy (Karakousis 2009). The emergence of multidrug-resistant TB (MDR-TB), defined as resistance to the first-line drugs isoniazid and rifampicin, and extensively drugresistant TB (XDR-TB), defined as MDR-TB with additional resistance to fluoroquinolones and at least one of the injectable second-line drugs (capreomycin, kanamycin, and amikacin), poses formidable challenges to global TB control efforts. The high global incidence of drug-resistant TB, estimated annually to be $\sim 500,000$ cases of MDR-TB of which $5 \%$ to $7 \%$ represent $\mathrm{XDR}-\mathrm{TB}$, underscores the need to understand the molecular mechanisms of drug resistance, with the ultimate goals of developing new techniques for rapid detection of drug resistance and identification of new drug targets.

This chapter presents an updated review of the mechanisms of action and resistance of the main old and new anti-tuberculosis agents.

\section{Intrinsic and acquired drug resistance}

Intrinsic resistance refers to the innate ability of a bacterium to resist the activity of a particular antimicrobial agent through its inherent structural or functional characteristics. Intrinsic drug resistance in $M$. tuberculosis has been attributed to its unique cell wall properties, including the presence of mycolic acids, which are high-molecular-weight a-alkyl, $\beta$-hydroxy fatty acids covalently attached to arabinogalactan, and which constitute a 
very hydrophobic barrier responsible for resistance to certain antibiotics (Karakousis, Bishai et al. 2004). In addition, $M$. tuberculosis possesses $\beta$-lactamase enzymes, which confer intrinsic resistance to $\beta$-lactam antibiotics, while efflux mechanisms appear to play an important role in resistance to antibiotics such as tetracycline and the aminoglycosides.

Acquired drug resistance occurs when a microorganism obtains the ability to resist the activity of a particular antimicrobial agent to which it was previously susceptible. Acquired drug resistance in $M$. tuberculosis is caused mainly by spontaneous mutations in chromosomal genes, and the selective growth of such drug-resistant mutants may be promoted during suboptimal drug therapy (Kochi, Vareldzis et al. 1993). The rate of genetic mutations leading to resistance varies somewhat among anti-tuberculosis drugs, from a frequency of $\sim 10-5-10-6$ organisms for isoniazid to $\sim 10-7-10-8$ organisms for rifampin (Karakousis 2009). Since the bacterial burden typically present in pulmonary cavities does not exceed $10^{12}$ bacilli (Canetti 1965), combination therapy is highly effective for drugsusceptible disease, and the risk for development of acquired drug resistance is minimized.

\section{Old TB drugs}

\subsection{Isoniazid}

Isoniazid (isonicotinic acid hydrazide, INH) has been the most commonly used antituberculosis since recognition of its clinical activity in 1952 (Robitzek and Selikoff 1952). Consisting of a pyridine ring and a hydrazide group, INH is a nicotinamide analog, structurally related to the anti-tuberculosis drugs ethionamide and pyrazinamide. Because of its significant bactericidal activity, it has become a critical component of the first-line antituberculous regimens, although in the last two decades resistance to INH has been reported with increasing frequency.<smiles>NNC(=O)c1ccncc1</smiles>

Isoniazid

\subsubsection{Mechanism of action}

INH appears to penetrate host cells readily (Mackaness and Smith 1952) and diffuses across the M. tuberculosis membrane (Suter 1952; Bardou, Raynaud et al. 1998). INH is a pro-drug, requiring oxidative activation by the $M$. tuberculosis catalase-peroxidase enzyme KatG (Zhang, Heym et al. 1992). Although the active metabolites of INH have been reported to inhibit multiple essential cellular pathways, including synthesis of nucleic acids (Gangadharam, Harold et al. 1963), phospholipids (Brennan, Rooney et al. 1970), and NAD metabolism (Zatman, Kaplan et al. 1954; Bekierkunst 1966), the primary pathway 
responsible for the killing activity of the drug is mycolic acid synthesis (Winder and Collins 1970; Takayama, Wang et al. 1972; Takayama, Schnoes et al. 1975). Thus, the activated form of the drug binds tightly to the NADH-dependent enoyl acyl carrier protein (ACP) reductase InhA (Banerjee, Dubnau et al. 1994), a component of the fatty acid synthase II system of mycobacteria, which is essential for fatty acid elongation (Quemard, Sacchettini et al. 1995). INH does not directly interact with InhA, as X-ray crystallographic and mass spectrometry data revealed that the activated form of INH covalently attaches to the nicotinamide ring of NAD bound within the active site of InhA, causing NADH to dissociate from InhA (Dessen, Quemard et al. 1995; Rozwarski, Grant et al. 1998). However, the precise mechanism by which INH kills M. tuberculosis remains to be elucidated.

\subsubsection{Mechanism of resistance}

Because INH is the most commonly used antituberculosis drug, resistance to INH occurs more frequently among clinical isolates than resistance to any other agent (Karakousis 2009).

Mutations in INH-resistant clinical isolates are most commonly detected in the kat G gene, occurring in $50-80 \%$ of cases, thus reducing the ability of the catalase-peroxidase to activate the INH pro-drug. The katG gene is located in a highly variable and unstable region of the $M$. tuberculosis genome, with missense and nonsense mutations, insertions, deletions, truncation and, more rarely, full gene deletions observed. Depending on the type of mutation, and the degree to which function of the KatG enzyme is preserved, the ensuing minimum inhibitory concentration (MIC) of isoniazid may range from 0.2 to 256 $\mathrm{mg} / \mathrm{L}$. Point mutations in katG are more commonly observed than other types of mutations, and a single point mutation resulting in substitution of threonine for serine at residue 315 (S315T) accounts for the majority of INH resistance among clinical isolates (Marttila, Soini et al. 1998; Abate, Hoffner et al. 2001). The S315T mutation results in a significant reduction in catalase and peroxidase activity, and is associated with high-level INH resistance $(\mathrm{MIC}=5-10 \mu \mathrm{g} / \mathrm{mL}$ ) (Rouse, DeVito et al. 1996; Saint-Joanis, Souchon et al. 1999).

INH resistance may also arise from mutations in inhA, resulting in reduced affinity of the enzyme for NADH without affecting its enoyl reductase activity (Basso, Zheng et al. 1998), or in the promoter region of the mabAinhA operon (Musser, Kapur et al. 1996), resulting in overexpression of the wild-type enzyme. Generally, mutations in inhA or in the promoter region of its operon usually confer low-level resistance (MIC $=0.2-1 \mathrm{mg} / \mathrm{L}$ ) (Wade and Zhang 2004). In addition to conferring resistance to INH, mutations in inhA also cause resistance to the structurally related second-line drug ethionamide.

Mutations in the $n d h$ gene, which encodes a NADH dehydrogenase, confer resistance to INH and ethionamide in M. smegmatis (Miesel, Weisbrod et al. 1998), and have been detected in INH-resistant $M$. tuberculosis clinical isolates, which lack mutations in the kat $G$ or inhA genes (Lee, Teo et al. 2001). Defective NADH dehydrogenase could lead to an increased ratio of $\mathrm{NADH} / \mathrm{NAD}$, thereby interfering with KatG-mediated peroxidation of INH, or by displacing the INH/NAD adduct from the InhA active site (Miesel, Weisbrod et al. 1998). Furthermore, mutations in kasA and $\operatorname{ahpC}$ genes have been associated with INH resistance. Nevertheless, as many as a quarter of all clinical INH-resistant isolates do not 
have mutations in any of the above genes, suggesting alternative mechanisms of INH resistance (Karakousis 2009).

\subsection{Rifampin and other rifamycins}

The rifamycins were first isolated in 1957 from Amycolatopsis (formerly Streptomyces) mediterranei as part of an Italian antibiotic screening program (Sensi 1983). Their incorporation into the standard anti-tuberculosis regimen allowed reduction of the duration of treatment from 18 to 9 months. Although the early bactericidal activity of the rifamycins is inferior to that of $\mathrm{INH}$, the former are the most potent sterilizing agents available in TB chemotherapy, continuing to kill persistent tubercle bacilli throughout the duration of therapy (Mitchison 1985; Grosset, Lounis et al. 1998). Rifampin is a broad-spectrum antibiotic and the most widely used rifamycin to treat TB.

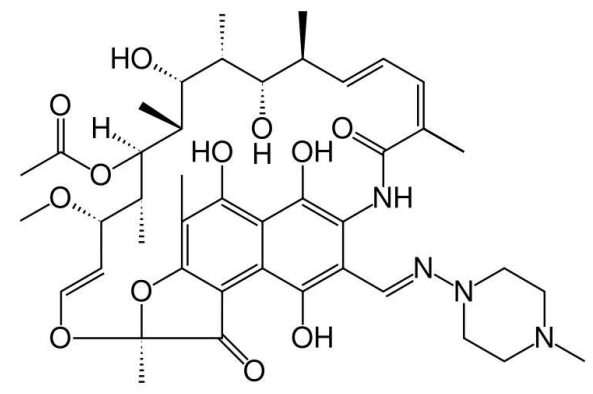

Rifampicin

\subsubsection{Mechanism of action}

Rifamycins contain an aromatic nucleus linked on both sides by an aliphatic bridge. The rifamycins easily diffuse across the $M$. tuberculosis cell membrane due to their lipophilic profile (Wade and Zhang 2004). Their bactericidal activity has been attributed to their ability to inhibit transcription by binding with high affinity to bacterial DNA-dependent RNA polymerase. Although the molecular target of rifampin has been well characterized, the precise mechanism by which this interaction leads to mycobacterial killing remains unclear.

\subsubsection{Mechanism of resistance}

Although INH monoresistance is relatively common in M. tuberculosis, resistance to rifampin alone is rare, and more than $90 \%$ of rifampin-resistant isolates are also resistant to INH. Therefore, rifampin resistance has been used as a surrogate marker for MDR-TB. Resistance to rifampin in $M$. tuberculosis arises at a frequency of $10^{-7}$ to $10^{-8}$ organisms, most commonly as single point mutations in the $r p o B$ gene, which encodes the $\beta$-subunit of RNA polymerase (Telenti, Imboden et al. 1993). In over $90 \%$ of rifampin-resistant clinical isolates, point mutations cluster in an 81-base pair "hot-spot" region between codons 507 and 533 of the $r p o B$ gene, with mutations in codons 531 [Ser] and 526 [His] predominating (Ramaswamy and Musser 1998). However, a small percentage of rifampin-resistant isolates 
$(<5 \%)$ do not contain any mutations in the $r p o B$ gene, suggesting alternative resistance mechanisms, potentially including altered rifampin permeability or mutations in other RNA polymerase subunits (Musser 1995).

Higher doses of the rifamycins, especially rifapentine, have the potential to further shorten the duration of TB treatment. Therefore, there is renewed interest in establishing the maximally tolerated dose of these drugs, and a number of clinical trials are planned or underway to examine the safety, pharmacokinetics and efficacy of higher than standard doses of rifampicin or rifapentine in first-line TB treatment (Ginsberg 2010).

\subsection{Pyrazinamide}

Since the discovery of pyrazinamide (PZA) in 1952 (Yeager, Munroe et al. 1952), and its routine use to treat $\mathrm{TB}$, the duration of treatment required to achieve acceptable relapse rates has been reduced from 9-12 months to the current 6 months (Steele and Des Prez 1988). Although its bactericidal activity is inferior to that of INH and rifampin (Jindani, Aber et al. 1980), the potent sterilizing activity and treatment-shortening potential of PZA has been attributed to the drug's unique ability to target semi-dormant populations of bacilli residing within an acidic environment (Mitchison 1985).

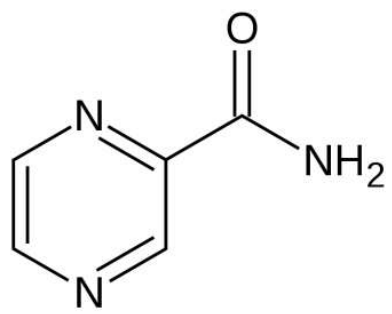

Pyrazinamide

\subsubsection{Mechanism of action}

PZA is an amide derivative of pyrazine-2-carboxylic acid and nicotinamide analog. Despite recognition of its anti-tuberculosis activity six decades ago, the mechanism of action of PZA remains poorly understood. PZA has been hypothesized to act against bacilli residing in acidified compartments of the lung that are present during the early inflammatory stages of infection (Mitchison 1985), since the drug's sterilizing activity appears to be limited to the first 2 months of therapy $(1986 ; 1986 ; 1991)$. PZA enters tubercle bacilli passively and via an ATP-dependent transport system (Raynaud, Laneelle et al. 1999). Intracellular accumulation of the drug occurs because of an inefficient efflux system unique to M. tuberculosis. PZA, like $\mathrm{INH}$, is a pro-drug, requiring activation to its active form, pyrazinoic acid (POA), by the enzyme pyrazinamidase (PZase) (Konno, Feldmann et al. 1967; Scorpio and Zhang 1996). Uptake and intrabacillary accumulation of POA is enhanced when the extracellular $\mathrm{pH}$ is acidic (Zhang, Scorpio et al. 1999). The anti-tuberculosis activity of PZA has been attributed to disruption of the proton motive force required for essential membrane transport functions by POA at acidic $\mathrm{pH}$ (Zhang, Wade et al. 2003), although investigation into potential specific cellular targets is ongoing. 


\subsubsection{Mechanism of resistance}

PZA resistance has been attributed primarily to mutations in the $p n c A$ gene encoding PZase (Scorpio and Zhang 1996). Most mutations, including point mutations, deletions, and insertions, have been reported in a 561-bp region of the open reading frame or in an 82-bp region of its putative promoter (Scorpio, Lindholm-Levy et al. 1997; Jureen, Werngren et al. 2008). The relatively high degree of diversity in pncA mutations among PZA-resistant clinical isolates has complicated the development of molecular assays for the rapid and economical detection of PZA resistance. A small percentage of isolates with high-level PZA resistance contain no mutations in $p n c A$ or its promoter, suggesting alternative mechanisms of resistance such as deficient uptake (Raynaud, Laneelle et al. 1999), enhanced efflux, or altered $p n c A$ regulation.

The high specificity of PZA for M. tuberculosis, with little or no activity against M. bovis and other mycobacteria, is attributable to $p n c A$ mutations, which render PZase inactive in the latter mycobacterial species.

\subsection{Ethambutol}

Ethambutol (EMB; dextro-2,2.-(ethylenediimino)-di-1-butanol) was initially reported to have anti-tuberculosis activity in 1961 and, together with INH, rifampin, and PZA, constitutes the modern-day short-course for the treatment of drug-susceptible TB. Like INH, EMB primarily kills actively multiplying bacilli and has very poor sterilizing activity.

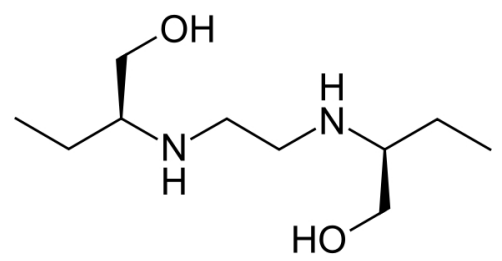

Ethambutol

\subsubsection{Mechanism of action}

The primary pathway affected by EMB appears to be that of arabinogalactan biosynthesis through inhibition of cell wall arabinan polymerization (Mikusova, Slayden et al. 1995). EMB also has been reported to inhibit several other cellular pathways, including RNA metabolism (Forbes, Kuck et al. 1962; Forbes, Kuck et al. 1965), transfer of mycolic acids into the cell wall (Takayama, Armstrong et al. 1979), phospholipid synthesis (Cheema and Khuller 1985; Cheema and Khuller 1985), and spermidine biosynthesis (Paulin, Brander et al. 1985).

\subsubsection{Mechanism of resistance}

Resistance to EMB in M. tuberculosis is usually associated with point mutations in the embCAB operon (Belanger, Besra et al. 1996). Genetic and biochemical studies have shown that the EmbA and EmbB proteins are involved in the formation of the proper terminal hexaarabinofuranoside motif during arabinogalactan synthesis (Escuyer, Lety et al. 2001), 
while EmbC is involved in lipoarabinomannan synthesis (Zhang, Torrelles et al. 2003). As the majority of EMB-resistant clinical isolates contain mutations in the embB gene (Sreevatsan, Stockbauer et al. 1997; Telenti, Philipp et al. 1997; Ramaswamy, Amin et al. 2000), EmbB is considered to be the main target of EMB, although X-ray crystallographic data supporting this interaction are lacking. More recently, the most commonly observed mutations in $e m b B$ codon 306 have been reported to be associated with variable degrees of EMB resistance, indicating that such mutations may be necessary but not sufficient for highlevel EMB resistance. Other potential mutations involved in EMB resistance include a Gln379Arg substitution in $M$. tuberculosis embR, as well as mutations in the $r m l D, r m l A 2$, and Rv0340 genes. As many as one quarter of all EMB-resistant $M$. tuberculosis isolates do not harbor mutations in any of the genes described above, suggesting alternative mechanisms of EMB resistance (Karakousis 2009).

\subsection{Aminoglycosides}

The discovery of streptomycin in the early 1940s represented the first breakthrough in TB chemotherapy. Although relapse rates are comparable when streptomycin is substituted for EMB as the fourth drug in addition to INH, rifampin, and PZA, the poor oral absorption of streptomycin, which necessitates parenteral administration, as well as the toxicity profile of the aminoglycosides, have favored the use of EMB in first-line anti-tuberculosis therapy. Other aminoglycosides with significant antimycobacterial activity include kanamycin and amikacin. Aminoglycosides are used currently as second-line drugs primarily in the treatment of MDR-TB.

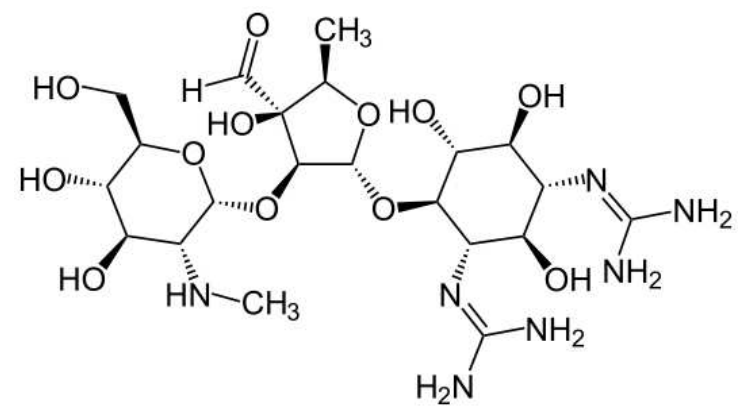

Streptomycin

\subsubsection{Mechanism of action}

As in other bacteria, the mode of action of the aminoglycosides against mycobacterial species is through their binding to the $30 \mathrm{~S}$ ribosomal subunit, which affects polypeptide synthesis, ultimately resulting in inhibition of translation.

\subsubsection{Mechanism of resistance}

Resistance to streptomycin and the other aminoglycosides in M. tuberculosis usually develops by mutation of the ribosome target binding sites. Interestingly, although crossresistance is observed between amikacin and kanamycin (Allen, Mitchison et al. 1983), these drugs are not cross-resistant with streptomycin (Tsukamura and Mizuno 1975), suggesting 
distinct mechanisms of resistance. Mutations in the rpsL gene, which encodes the ribosomal protein S12, account for approximately half of all streptomycin-resistant clinical isolates (Nair, Rouse et al. 1993; Cooksey, Morlock et al. 1996), with the K43R mutation predominating. In about $20 \%$ of streptomycin-resistant $M$. tuberculosis clinical isolates, such resistance is associated with mutations in the rrs gene, which are usually clustered in the regions surrounding nucleotides 530 or 912 (Douglass and Steyn 1993; Cooksey, Morlock et al. 1996). The particular vulnerability of rrs to mutation, leading to streptomycin resistance in M. tuberculosis and other slow-growing mycobacteria, can be explained by the fact that these mycobacterial species, unlike other bacteria, contain only a single copy of the rrs gene. Generally, mutations in the rpsL and rrs genes confer high-level (MIC > 1,000 mg/L) or intermediate-level $(\mathrm{MIC}=64-512 \mathrm{mg} / \mathrm{L}$ ) resistance to streptomycin (Sreevatsan, Pan et al. 1996). On the other hand, mechanisms of low-level resistance to streptomycin (MIC $=4-32$ $\mathrm{mg} / \mathrm{L})$ remain largely undefined but may be attributable to changes in cell envelope permeability or diminished drug uptake (Honore and Cole 1994; Cooksey, Morlock et al. 1996). More recently, it has been shown that mutations in gidB, which encodes a conserved S-adenosylmethionine-dependent 16S rRNA methyltransferase, can confer low-level resistance to streptomycin (Wong, Lee et al. 2011).

\subsection{Fluoroquinolones}

The fluoroquinolones (moxifloxacin, gatifloxacin, sparfloxacin, levofloxacin, ofloxacin, and ciprofloxacin), are bactericidal antibiotics with excellent activity against $M$. tuberculosis and are currently used as second-line drugs in TB treatment. New-generation fluoroquinolones [moxofloxacin, gatifloxacin] are under clinical evaluation as first-line antibiotics with the goal of shortening the duration of TB treatment.<smiles>O=C(O)c1cn(C2CC2)c2cc(N3CCNCC3)c(F)cc2c1=O</smiles>

Ciprofloxacin<smiles>COc1c(N2C[C@H]3NCCC[C@@H]3C2)c(F)cc2c(=O)c(C(=O)O)cn(C3CC3)c12</smiles>

Moxifloxacin

\subsubsection{Mechanism of action}

Fluoroquinolones exert their powerful antibacterial activity by trapping gyrase and topoisomerase IV on DNA as ternary complexes, thereby blocking the movement of replication forks and transcription complexes (Drlica and Malik 2003). Unlike most other bacterial species, $M$. tuberculosis lacks topoisomerase IV, but contains the genes gyrA and gyrB encoding the A and B subunits, respectively, of DNA gyrase (Cole, Brosch et al. 1998).

\subsubsection{Mechanism of resistance}

Fluoroquinolone resistance in $M$. tuberculosis is most commonly associated with mutations in the conserved quinolone resistance-determining region (QRDR) of gyrA and gyrB involved in the interaction between the drug and DNA gyrase (Ginsburg, Grosset et al. 2003). The degree of fluoroquinolone resistance is dictated by the specific amino acid 
substitution in the QRDR, as well as the number of resistance mutations present. Therefore, while individual mutations in gyrA may confer low-level resistance (MIC $>2 \mathrm{mg} / \mathrm{L}$ ) (Kocagoz, Hackbarth et al. 1996), high-level resistance to fluoroquinolones usually requires multiple mutations in gyrA, or concurrent mutations in gyrA and gyrB (Kocagoz, Hackbarth et al. 1996; Xu, Kreiswirth et al. 1996). The most frequently observed mutations associated with fluoroquinolone resistance in M. tuberculosis are at positions Ala-90 and Asp-94 in the gyrA gene. Codon 95 contains a naturally occurring polymorphism (Ser or Thr) that is not related to fluoroquinolone resistance, as it occurs in both fluoroquinolone-susceptible and fluoroquinolone-resistant strains. Interestingly, mutations at position 80 of gyrA have been reported to cause hypersusceptibility to fluoroquinolones, especially when present with other resistance mutations (Aubry, Veziris et al. 2006). Since mutations in the QRDR region of gyrA are identified in only $42-85 \%$ of fluoroquinolone-resistant clinical isolates, alternative mechanisms of resistance are presumed to exist, including the potential participation of efflux pumps. Although full cross-resistance is commonly observed across fluoroquinolones, von Groll et al. (Von Groll, Martin et al. 2009) recently reported a strain with an Asn-533-Thr mutation in gyrB that was resistant to moxifloxacin and gatifloxacin but susceptible to ofloxacin.

\subsection{Macrolides}

\subsubsection{Mechanism of action}

The macrolides are broad-spectrum antibiotics, which exert their antibacterial effect by binding to the bacterial $50 \mathrm{~S}$ ribosomal subunit and inhibiting RNA-dependent protein synthesis (Piscitelli, Danziger et al. 1992). However, these drugs have limited activity against wild-type M. tuberculosis (Bermudez and Yamazaki 2004). The possible role of macrolides in TB treatment is an area of active investigation, as recent studies have reported synergy of macrolides in combination with other antibiotics.

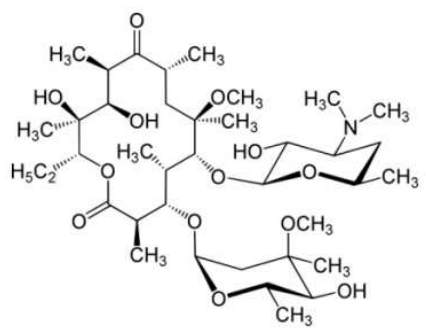

Clarithromycin

\subsubsection{Mechanism of resistance}

Intrinsic resistance to the macrolides in $M$. tuberculosis has been attributed to low cell wall permeability and expression of the erm(37) gene encoding a 23S rRNA methyltransferase, which is present in all members of the $M$. tuberculosis complex but absent in nontuberculous mycobacteria (Buriankova, Doucet-Populaire et al. 2004). Subinhibitory concentrations of clarithromycin have been shown to cause induction of erm(37) expression and a 4- to 8-fold increase in MIC (Andini and Nash 2006). 


\subsection{Ethionamide}

Ethionamide, a synthetic compound structurally related to $\mathrm{INH}$, is a pro-drug, requiring activation by the monooxygenase EthA (Baulard, Betts et al. 2000; DeBarber, Mdluli et al. 2000; Vannelli, Dykman et al. 2002). Similar to INH, ethionamide inhibits mycolic acid synthesis by binding the ACP reductase InhA. Approximately three-quarters of $M$. tuberculosis isolates with high-level ethionamide resistance (MIC $>50 \mathrm{mg} / \mathrm{L}$ ) have mutations in ethA or inhA (Morlock, Metchock et al. 2003). Recently, other potential mechanisms of resistance have been identified, as $M$. tuberculosis $m s h A$ deletion mutants were found to be defective in mycothiol biosynthesis and resistant to ethionamide, likely due to defective activation of the drug (Vilcheze, Av-Gay et al. 2008).

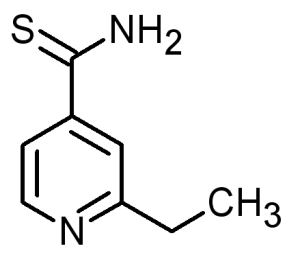

Ethionamide

\subsection{Capreomycin}

Capreomycin is a macrocyclic polypeptide antibiotic isolated from Streptomyces capreolus (Karakousis 2009). Capreomycin, like streptomycin and kanamycin, inhibits protein synthesis through modification of ribosomal structures at the 16S rRNA (Wade and Zhang 2004). Recent studies using site-directed mutagenesis have identified the binding site of capreomycin on 16S rRNA helix 44 (Akbergenov, Shcherbakov et al. 2011). In M. tuberculosis, resistance to capreomycin and kanamycin has been associated with mutations in the rrs gene encoding 16S rRNA (Taniguchi, Chang et al. 1997; Alangaden, Kreiswirth et al. 1998). Mutations in the gene tlyA encoding a 2'-O-methyltransferase of $16 \mathrm{~S}$ rRNA and 23S rRNA have been implicated in resistance to capreomycin and viomycin (Johansen, Maus et al. 2006), and such resistance is generally associated with the addition of methyl groups to rRNA rather than their loss (Sander, Meier et al. 1996). However, recent studies have shown that capreomycin-resistant strains lack mutations in tlyA (Jugheli, Bzekalava et al. 2009).<smiles>NCCCC(N)CC(=O)NCC(NC(=O)C(CO)NC(=O)C(N)CNC(=O)C(NC(=O)/C(=C/NC(N)=O)NC(=O)C(N)CCCN)C1CC=NC(N)N1)C(=O)NC(N)=O</smiles>

Capreomycin 


\subsection{Cycloserine}

Cycloserine is a d-alanine analogue, which interrupts peptidoglycan synthesis by inhibiting the enzymes d-alanine racemase (AlrA) and d-alanine:d-alanine ligase (Ddl) (Caceres, Harris et al. 1997). Overexpression of M. tuberculosis AlrA and Ddl on a multicopy vector results in resistance to D-cycloserine in M. smegmatis and M. bovis BCG (Caceres, Harris et al. 1997; Feng and Barletta 2003), although whether similar mechanisms are responsible for cycloserine resistance in $M$. tuberculosis remain to be determined.

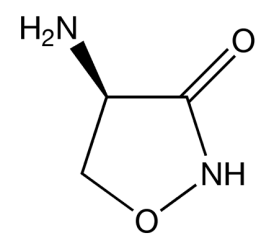

Cycloserine

\subsection{Paraaminosalicylic acid}

Paraaminosalicylic acid (PAS) is thought to inhibit folic acid biosynthesis and uptake of iron (Wade and Zhang 2004). Mutations in the thyA gene encoding the enzyme thimidylate synthesis of the folate biosynthesis pathway have been identified in PAS-resistant M. tuberculosis clinical isolates, suggesting that PAS may act as a folate antagonist (Rengarajan, Sassetti et al. 2004). However, only slightly more than a third of the evaluated PAS-resistant strains had mutations in thy A, suggesting the existence of additional mechanisms of PAS resistance. Thr202Ala has been reported as the most common mutation associated with PAS resistance, although this mutation has also been identified in several PAS-susceptible isolates (Leung, Yip et al. 2010).<smiles>Nc1ccc(C(=O)O)c(O)c1</smiles>

Paraaminosalicylic acid

\section{New TB drugs}

Several new drugs have emerged recently as potential candidates for the treatment of TB. In most cases, their mechanism of action is distinct from that of the classical anti-TB drugs, although strains resistant to several of the novel drugs already have been described even prior to their routine clinical use. 


\subsection{Nitroimidazoles}

Reduced oxygen tension may be an important microenvironmental condition encountered by persistent bacilli within necrotic lung granulomas in the human host (Haapanen, Kass et al. 1959). Interestingly, although in vitro exposure to microaerophilic conditions renders $M$. tuberculosis less susceptible to killing by INH and rifampin, the bacilli become susceptible to metronidazole (Wayne and Sramek 1994; Wayne and Hayes 1996) a nitroimidazole drug used to treat anaerobic infections. Metronidazole, which becomes reductively activated by the pyruvate:ferredoxin oxidereductase system under anoxic conditions (Edwards 1993) lacks antituberculous activity in mouse models (Brooks, Furney et al. 1999; Klinkenberg, Sutherland et al. 2008) and in guinea pigs (Hoff, Caraway et al. 2008), but displays activity in M. tuberculosis-infected rabbits (Via, Lin et al. 2008). Clinical studies evaluating the activity of metronidazole against MDR-TB are ongoing.

\subsubsection{PA-824}

PA-824, a small molecule nitroimidazopyran related to metronidazole, exhibits bactericidal activity against actively multiplying and stationary-phase cultures of $M$. tuberculosis, as well as in murine and guinea pig models of TB infection (Stover, Warrener et al. 2000; Lenaerts, Gruppo et al. 2005). In addition, PA-824 is highly active against multidrug-resistant clinical isolates of $M$. tuberculosis (MIC $<1 \mu \mathrm{g} / \mathrm{mL}$ ), suggesting no cross-resistance with current antituberculosis drugs (Lenaerts, Gruppo et al. 2005). Like metronidazole, PA-824 is a pro-drug requiring reductive activation of an aromatic nitro group, which involves an F420dependent glucose-6-phosphate dehydrogenase encoded by Rv0407 (fgd1) (Stover, Warrener et al. 2000) and deazaflavin-dependent nitroreductase (Ddn) encoded by Rv3547 (Singh, Manjunatha et al. 2008), in order to exert its antitubercular effect. The activity of PA-824 is at least partially mediated through inhibition of the oxidation of hydroxymycolates to ketomycolates, a terminal step in mycolic acid synthesis (Stover, Warrener et al. 2000). Recently, formation of the des-nitroimidazole metabolite of PA-824 was shown to generate reactive nitrogen species, including nitric oxide, which appears to contribute to the killing activity of PA-824 and may explain the activity of the drug against non-replicating bacilli (Singh, Manjunatha et al. 2008). Similar to INH, resistance to PA-824 is most commonly mediated by mutations that lead to loss of pro-drug activation, including those in the genes Rv0407 and Rv3547 encoding the activating enzymes.

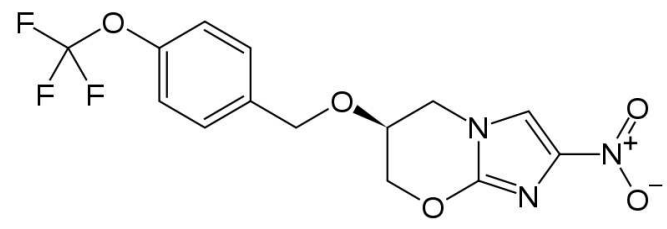

$P A-824$

\subsubsection{OPC-67683}

OPC-67683 is a nitro-dihydro-imidazooxazole derivative with potent activity against drugsusceptible M. tuberculosis and MDR-TB. The drug exerts its killing activity by inhibiting the synthesis of methoxy- and keto-mycolic acids. The substitution of OPC-67683 for INH and 
EMB in the standard regimen alongside rifampin and PZA led to more rapid sterilization of $M$. tuberculosis-infected mouse lungs (Matsumoto, Hashizume et al. 2006). Like the other nitroimidazoles, OPC-67683 is a pro-drug requiring reductive activation by $M$. tuberculosis. As in the case of PA-824, mutations in the Rv3547 gene have been identified in strains resistant to OPC-67683, indicating defective drug activation (Matsumoto, Hashizume et al. 2006).

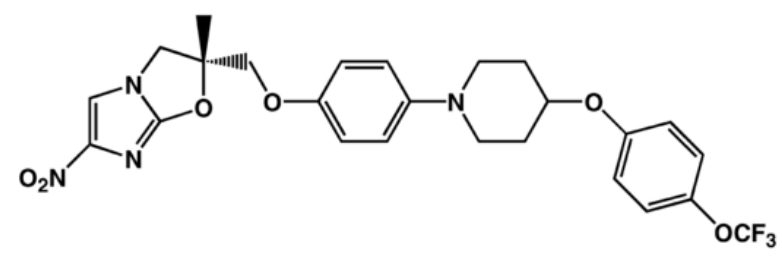

OPC-67683

\subsection{TMC207}

TMC207 (also named R207910 or "J compound") is a first-in-class anti-TB diarylquinoline with bactericidal and sterilizing activities against drug-susceptible and drug-resistant $M$. tuberculosis in vitro and in animal models, including in a murine model of latent TB infection (Zhang, Li et al. 2011). Mouse model studies suggest a synergistic relationship between TMC207 and PZA (Matteelli, Carvalho et al. 2010). TMC207 inhibits ATP synthase, a critical enzyme in the synthesis of ATP for M. tuberculosis (Andries, Verhasselt et al. 2005). The addition of TMC207 to standard therapy for MDR-TB significantly reduced the time to conversion to a negative sputum culture and increased the proportion of patients with conversion of sputum culture as compared with placebo (Diacon, Pym et al. 2009). Resistance to TMC207 is mediated by mutations in the atpE gene encoding the transmembrane and oligomeric C subunit of ATP synthase, typically at positions 63 or 66 (Petrella, Cambau et al. 2006). However, more recent studies have shown that a majority of in vitro-generated mutants resistant to TMC207 lacked mutations in atpE, indicating alternative mechanisms of drug resistance (Huitric, Verhasselt et al. 2010).<smiles>COc1nc2ccc(Br)cc2cc1[C@@H](c1ccccc1)[C@@](O)(CCN(C)C)c1cccc2ccccc12</smiles>

TMC207

\subsection{SQ109}

SQ109 was identified by screening a large synthesized combinatorial library based on the 1,2-ethylenediamine structure of EMB, and was found to have limited toxicity and potent 
activity against intracellular bacilli as well as in a murine model of chronic TB infection (Protopopova, Hanrahan et al. 2005). Early clinical data reveal the drug's potential to enhance the treatment of TB during the first 2 months of intensive therapy and also to treat MDR-TB (Lalloo and Ambaram 2010). Whether upregulation of $a h p C$ expression, observed in strains resistant to INH, EMB, and SQ109, plays a role in resistance to SQ109 or merely reflects a compensatory metabolic mechanism remains to be determined (Jia, Coward et al. 2005).<smiles>CC(C)=CCC/C(C)=C/CNCCNC1C2CC3CC(C2)CC1C3</smiles>

SQ109

\subsection{Phenothiazines}

The antipsychotic phenothiazine drug thioridazine has been reported to be active against drug-susceptible and drug-resistant $M$. tuberculosis, both in macrophages (Ordway, Viveiros et al. 2003) as well as in murine models (van Soolingen, Hernandez-Pando et al. 2010). Although serum concentrations above the MIC for M. tuberculosis (8-16 mg/L range) cannot be safely attained in humans, thioridazine still has potential as an antimycobacterial drug because of intracellular accumulation, such that concentrations inside macrophages are at least 10-fold higher than in serum. Despite the favorable toxicity profile of thioridazine relative to chlorpromazine and other phenothiazines, cardiac arrhythmia associated with prolongation of the QTc interval remains a risk. Thioridazine has been used successfully to cure patients with XDR-TB in Argentina and as salvage therapy in similar patients in India (Amaral, Boeree et al. 2010). The mechanism of action of thioridazine is likely multifactorial, as the drug appears to act on enzymes involved in fatty acid metabolism and membrane proteins, particularly efflux pumps (Dutta, Mazumdar et al. 2011), in addition to inhibiting type II NADH:menaquinone oxidoreductase as a phenothiazine (Weinstein, Yano et al. 2005). Mechanisms of M. tuberculosis resistance to the phenothiazines remain to be elucidated.<smiles>CSc1ccc2c(c1)N(CC[C@@H]1CCCCN1C)c1ccccc1S2</smiles>

Thioridazine

\subsection{Oxazolidinones}

Oxazolidinones are a new chemical class of synthetic antibiotics related to cycloserine with broad-spectrum activity against gram-positive pathogens through inhibition of protein synthesis. 


\subsubsection{Linezolid}

Linezolid is the first compound belonging to the oxazolidinone class approved for clinical use. Due to its ability to penetrate macrophages, linezolid is active against intracellular bacilli, exerting its activity by binding to the ribosomal $50 \mathrm{~S}$ subunit and thus inhibiting an early step in protein synthesis (Zhang 2005). Linezolid is most commonly used to treat drugresistant TB, but its use has been limited by toxicity concerns, particularly hematological disturbances such as leukopenia and thrombocytopenia, as well as peripheral neuropathy, which may be irreversible. While resistance to linezolid in M. tuberculosis clinical isolates is rarely reported, in vitro-selected mutants with high-level resistance to linezolid (MIC = 16$32 \mathrm{mg} / \mathrm{L}$ ) have been found to contain mutations at G2061T and G2576T in the 23S rRNA gene (Hillemann, Rusch-Gerdes et al. 2008). On the other hand, mutants with lower level linezolid resistance $(\mathrm{MIC}=4-8 \mathrm{mg} / \mathrm{L}$ ) lack mutations in the $23 \mathrm{~S}$ rRNA gene, implicating other possible mechanisms of resistance.

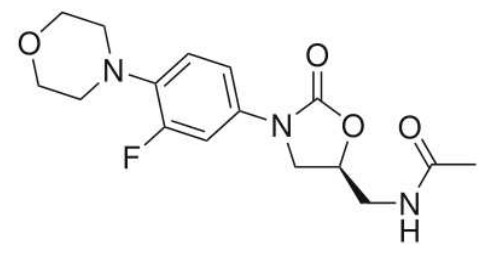

Linezolid

\subsubsection{PNU-100480}

PNU-100480, another oxazolidinone, has been shown to have more potent activity against M. tuberculosis than linezolid, as the MIC of PNU100480 is half that of linezolid, and is as bactericidal as isoniazid in an acute model of TB infection in mice (Cynamon, Klemens et al. 1999). Recent studies in the mouse model have shown that the addition of PNU-100480 to the standard first-line regimen of rifampin, INH, and PZA can shorten the duration of treatment necessary to prevent relapse (Williams, Brickner et al. 2009), suggesting that this drug may have sterilizing activity against drug-susceptible and drug-resistant M. tuberculosis. Recent Phase I studies have shown that PNU-100480 is safe and well tolerated at all tested doses, and exhibits synergy with PZA in an ex vivo whole-blood culture assay (Wallis, Jakubiec et al. 2011). Resistance mechanisms are expected to be similar to those of linezolid.

\subsubsection{AZD5847}

AZD5847 was originally designed for treatment of gram-positive infections, but was later repositioned for TB treatment with the goal of improving the toxicity profile associated with linezolid, including inhibition of mitochondrial protein synthesis, thrombocytopenia, and myelosuppression (Koul, Arnoult et al. 2011). Like linezolid, AZD5847 has bactericidal activity against $M$. tuberculosis in macrophages, as well as in murine models of acute and chronic TB infection. Recent Phase I trials revealed that oral administration of the drug up to $800 \mathrm{mg}$ twice daily for 14 days was satisfactorily tolerated in healthy volunteers. Although 
bioavailability decreases with increasing dose, this effect may be largely compensated if taken within 2 hours of meals, and the exposures achieved in man correspond to efficacious exposures in the mouse model of TB infection (B. Subramanian, Gordon Research Conference on Tuberculosis Drug Development, July 2011). Phase 2 studies to be conducted in South Africa are in the planning stage.

\subsection{Benzothiazinones}

The 1,3-benzothiazin-4-ones (BTZs) represent a new class of drugs, which have activity against M. tuberculosis in vitro, ex vivo, and in murine TB models (Makarov, Manina et al. 2009). BTZs are activated in M. tuberculosis by reduction of an essential nitro group to a nitroso derivative, which then specifically reacts with a cysteine residue in the active site of the enzyme decaprenylphosphoryl- $\beta$-D-ribose 2'-epimerase (DprE1) (Trefzer, RengifoGonzalez et al. 2010). Inhibition of this enzymatic activity abolishes the formation of decaprenylphosphoryl arabinose, a key precursor that is required for the synthesis of the cell-wall arabinans, thus causing bacterial lysis and death (Makarov, Manina et al. 2009). Although spontaneous BTZ-resistant laboratory mutants were found to have a Ser or Gly substitution at codon Cys387 of $d p r E 1$, resistance to BTZs has not been reported in clinical M. tuberculosis isolates (Pasca, Degiacomi et al. 2010). Recently, a novel resistance mechanism to BTZ was described in M. smegmatis involving the overexpression of the nitroreductase $\mathrm{NfnB}$, which leads to the inactivation of the drug by reduction of a critical nitro-group to an amino-group (Manina, Bellinzoni et al. 2010). However, M. tuberculosis seems to lack nitroreductases able to inactivate BTZs.

\section{Antibiotic tolerance}

Antibiotic tolerance refers to the ability of nonreplicating bacteria to resist killing by cell wall-active antibiotics, which target actively multiplying organisms (Tomasz, Albino et al. 1970). This phenomenon is distinct from drug resistance (intrinsic or acquired), since it is not attributable to genetic mutations, and the organisms regain susceptibility to these antibiotics once the stress conditions have been removed and bacterial growth resumes. The prolonged duration of antibiotic treatment required to eradicate TB is believed to reflect the altered physiological state of "persistent" bacilli, which have developed tolerance to standard anti-tuberculosis drugs, particularly to isoniazid, which inhibits mycolic acid synthesis (Karakousis, Williams et al. 2008). One of the major challenges facing current TB drug development programs is to identify compounds with sterilizing activity against antibiotic tolerant "persisters", with the ultimate goal of shortening the duration of TB treatment.

\section{Conclusion}

The principal etiology of drug-resistant $\mathrm{TB}$ remains inadequate and/or incomplete treatment, including poor medical adherence to the standard treatment regimen and the addition of a single active drug to a failing drug regimen (Sharma and Mohan 2006). Given the increasing global prevalence of drug-resistant $\mathrm{TB}$, it is of paramount importance to understand the mode of action of each drug as well as the molecular basis of drug resistance. Novel anti-TB drugs, which are safe, able to shorten the course of treatment, 
effective against drug-resistant strains and latent TB infection, are urgently needed, especially in the era of MDR- and XDR-TB.

\section{References}

(1986). "Controlled clinical trial of 4 short-course regimens of chemotherapy (three 6-month and one 8-month) for pulmonary tuberculosis: final report. East and Central African/British Medical Research Council Fifth Collaborative Study." Tubercle 67(1): 5-15.

(1986). "Long-term follow-up of a clinical trial of six-month and four-month regimens of chemotherapy in the treatment of pulmonary tuberculosis. Singapore Tuberculosis Service/British Medical Research Council." Am Rev Respir Dis 133(5): 779-783.

(1991). "Controlled trial of 2, 4, and 6 months of pyrazinamide in 6-month, three-timesweekly regimens for smear-positive pulmonary tuberculosis, including an assessment of a combined preparation of isoniazid, rifampin, and pyrazinamide. Results at 30 months. Hong Kong Chest Service/British Medical Research Council." Am Rev Respir Dis 143(4 Pt 1): 700-706.

Abate, G., S. E. Hoffner, et al. (2001). "Characterization of isoniazid-resistant strains of Mycobacterium tuberculosis on the basis of phenotypic properties and mutations in katG." Eur J Clin Microbiol Infect Dis 20(5): 329-333.

Akbergenov, R., D. Shcherbakov, et al. (2011). "Molecular basis for the selectivity of antituberculosis compounds capreomycin and viomycin." Antimicrob Agents Chemother.

Alangaden, G. J., B. N. Kreiswirth, et al. (1998). "Mechanism of resistance to amikacin and kanamycin in Mycobacterium tuberculosis." Antimicrob Agents Chemother 42(5): 1295-1297.

Allen, B. W., D. A. Mitchison, et al. (1983). "Amikacin in the treatment of pulmonary tuberculosis." Tubercle 64(2): 111-118.

Amaral, L., M. J. Boeree, et al. (2010). "Thioridazine cures extensively drug-resistant tuberculosis (XDR-TB) and the need for global trials is now!" Int J Antimicrob Agents 35(6): 524-526.

Andini, N. and K. A. Nash (2006). "Intrinsic macrolide resistance of the Mycobacterium tuberculosis complex is inducible." Antimicrob Agents Chemother 50(7): 2560-2562.

Andries, K., P. Verhasselt, et al. (2005). "A diarylquinoline drug active on the ATP synthase of Mycobacterium tuberculosis." Science 307(5707): 223-227.

Aubry, A., N. Veziris, et al. (2006). "Novel gyrase mutations in quinolone-resistant and hypersusceptible clinical isolates of Mycobacterium tuberculosis: functional analysis of mutant enzymes." Antimicrob Agents Chemother 50(1): 104-112.

Banerjee, A., E. Dubnau, et al. (1994). "inhA, a gene encoding a target for isoniazid and ethionamide in Mycobacterium tuberculosis." Science 263(5144): 227-230.

Bardou, F., C. Raynaud, et al. (1998). "Mechanism of isoniazid uptake in Mycobacterium tuberculosis." Microbiology 144 ( Pt 9): 2539-2544.

Basso, L. A., R. Zheng, et al. (1998). "Mechanisms of isoniazid resistance in Mycobacterium tuberculosis: enzymatic characterization of enoyl reductase mutants identified in isoniazid-resistant clinical isolates." Journal of Infectious Diseases 178(3): 769-775. 
Baulard, A. R., J. C. Betts, et al. (2000). "Activation of the pro-drug ethionamide is regulated in mycobacteria." J Biol Chem 275(36): 28326-28331.

Bekierkunst, A. (1966). "Nicotinamide-adenine dinucleotide in tubercle bacilli exposed to isoniazid." Science 152(721): 525-526.

Belanger, A. E., G. S. Besra, et al. (1996). "The embAB genes of Mycobacterium avium encode an arabinosyl transferase involved in cell wall arabinan biosynthesis that is the target for the antimycobacterial drug ethambutol." Proc Natl Acad Sci U S A 93(21): 11919-11924.

Bermudez, L. E. and Y. Yamazaki (2004). "Effects of macrolides and ketolides on mycobacterial infections." Curr Pharm Des 10(26): 3221-3228.

Brennan, P. J., S. A. Rooney, et al. (1970). "The lipids of Mycobacterium tuberculosis BCG: fractionation, composition, turnover and the effects of isoniazid." Ir J Med Sci 3(8): 371-390.

Brooks, J. V., S. K. Furney, et al. (1999). "Metronidazole therapy in mice infected with tuberculosis." Antimicrob Agents Chemother 43(5): 1285-1288.

Buriankova, K., F. Doucet-Populaire, et al. (2004). "Molecular basis of intrinsic macrolide resistance in the Mycobacterium tuberculosis complex." Antimicrob Agents Chemother 48(1): 143-150.

Caceres, N. E., N. B. Harris, et al. (1997). "Overexpression of the D-alanine racemase gene confers resistance to D-cycloserine in Mycobacterium smegmatis." Journal of Bacteriology 179(16): 5046-5055.

Canetti, G. (1965). "Present aspects of bacterial resistance in tuberculosis." Am Rev Respir Dis 92(5): 687-703.

Cheema, S. and G. K. Khuller (1985). "Metabolism of phospholipids in Mycobacterium smegmatis ATCC 607 in the presence of ethambutol." Indian J Med Res 82: 207-213.

Cheema, S. and G. K. Khuller (1985). "Phospholipid composition and ethambutol sensitivity of Mycobacterium smegmatis ATCC 607." Indian J Exp Biol 23(9): 511-513.

Cole, S. T., R. Brosch, et al. (1998). "Deciphering the biology of Mycobacterium tuberculosis from the complete genome sequence." Nature 393(6685): 537-544.

Cooksey, R. C., G. P. Morlock, et al. (1996). "Characterization of streptomycin resistance mechanisms among Mycobacterium tuberculosis isolates from patients in New York City." Antimicrob Agents Chemother 40(5): 1186-1188.

Cynamon, M. H., S. P. Klemens, et al. (1999). "Activities of several novel oxazolidinones against Mycobacterium tuberculosis in a murine model." Antimicrob Agents Chemother 43(5): 1189-1191.

DeBarber, A. E., K. Mdluli, et al. (2000). "Ethionamide activation and sensitivity in multidrug-resistant Mycobacterium tuberculosis." Proc Natl Acad Sci U S A 97(17): 9677-9682.

Dessen, A., A. Quemard, et al. (1995). "Crystal structure and function of the isoniazid target of Mycobacterium tuberculosis." Science 267(5204): 1638-1641.

Diacon, A. H., A. Pym, et al. (2009). "The diarylquinoline TMC207 for multidrug-resistant tuberculosis." N Engl J Med 360(23): 2397-2405.

Douglass, J. and L. M. Steyn (1993). "A ribosomal gene mutation in streptomycin-resistant Mycobacterium tuberculosis isolates." Journal of Infectious Diseases 167(6): 15051506. 
Drlica, K. and M. Malik (2003). "Fluoroquinolones: action and resistance." Curr Top Med Chem 3(3): 249-282.

Dutta, N. K., K. Mazumdar, et al. (2011). "New patentable use of an old neuroleptic compound thioridazine to combat tuberculosis: a gene regulation perspective." Recent Pat Antiinfect Drug Discov 6(2): 128-138.

Edwards, D. I. (1993). "Nitroimidazole drugs--action and resistance mechanisms. I. Mechanisms of action." J Antimicrob Chemother 31(1): 9-20.

Escuyer, V. E., M. A. Lety, et al. (2001). "The role of the embA and embB gene products in the biosynthesis of the terminal hexaarabinofuranosyl motif of Mycobacterium smegmatis arabinogalactan." J Biol Chem 276(52): 48854-48862.

Feng, Z. and R. G. Barletta (2003). "Roles of Mycobacterium smegmatis D-alanine:D-alanine ligase and D-alanine racemase in the mechanisms of action of and resistance to the peptidoglycan inhibitor D-cycloserine." Antimicrob Agents Chemother 47(1): 283291.

Forbes, M., N. A. Kuck, et al. (1962). "Mode of action of ethambutol." Journal of Bacteriology 84: 1099-1103.

Forbes, M., N. A. Kuck, et al. (1965). "Effect of Ethambutol on Nucleic Acid Metabolism in Mycobacterium Smegmatis and Its Reversal by Polyamines and Divalent Cations." Journal of Bacteriology 89: 1299-1305.

Gangadharam, P. R., F. M. Harold, et al. (1963). "Selective inhibition of nucleic acid synthesis in Mycobacterium tuberculosis by isoniazid." Nature 198: 712-714.

Ginsberg, A. M. (2010). "Drugs in development for tuberculosis." Drugs 70(17): 2201-2214.

Ginsburg, A. S., J. H. Grosset, et al. (2003). "Fluoroquinolones, tuberculosis, and resistance." Lancet Infectious Diseases 3(7): 432-442.

Grosset, J., N. Lounis, et al. (1998). "Once-weekly rifapentine-containing regimens for treatment of tuberculosis in mice." Am J Respir Crit Care Med 157(5 Pt 1): 14361440 .

Haapanen, J. H., I. Kass, et al. (1959). "Studies on the gaseous content of tuberculous cavities." Am Rev Respir Dis 80(1, Part 1): 1-5.

Hillemann, D., S. Rusch-Gerdes, et al. (2008). "In vitro-selected linezolid-resistant Mycobacterium tuberculosis mutants." Antimicrob Agents Chemother 52(2): 800801.

Hoff, D. R., M. L. Caraway, et al. (2008). "Metronidazole lacks antibacterial activity in guinea pigs infected with Mycobacterium tuberculosis." Antimicrob Agents Chemother 52(11): 4137-4140.

Honore, N. and S. T. Cole (1994). "Streptomycin resistance in mycobacteria." Antimicrob Agents Chemother 38(2): 238-242.

Huitric, E., P. Verhasselt, et al. (2010). "Rates and mechanisms of resistance development in Mycobacterium tuberculosis to a novel diarylquinoline ATP synthase inhibitor." Antimicrob Agents Chemother 54(3): 1022-1028.

Jia, L., L. Coward, et al. (2005). "Pharmacoproteomic effects of isoniazid, ethambutol, and Ngeranyl-N'-(2-adamantyl)ethane-1,2-diamine (SQ109) on Mycobacterium tuberculosis H37Rv." J Pharmacol Exp Ther 315(2): 905-911.

Jindani, A., V. R. Aber, et al. (1980). "The early bactericidal activity of drugs in patients with pulmonary tuberculosis." Am Rev Respir Dis 121(6): 939-949. 
Johansen, S. K., C. E. Maus, et al. (2006). "Capreomycin binds across the ribosomal subunit interface using tlyA-encoded 2'-O-methylations in $16 \mathrm{~S}$ and $23 \mathrm{~S}$ rRNAs." Mol Cell 23(2): 173-182.

Jugheli, L., N. Bzekalava, et al. (2009). "High level of cross-resistance between kanamycin, amikacin, and capreomycin among Mycobacterium tuberculosis isolates from Georgia and a close relation with mutations in the rrs gene." Antimicrob Agents Chemother 53(12): 5064-5068.

Jureen, P., J. Werngren, et al. (2008). "Pyrazinamide resistance and pncA gene mutations in Mycobacterium tuberculosis." Antimicrob Agents Chemother 52(5): 1852-1854.

Karakousis, P. C. (2009). Mechanisms of Action and Resistance of Antimycobacterial Agents. In: Antimicrobial Drug Resistance. D. L. Mayers, Springer: pp.271-291.

Karakousis, P. C., W. R. Bishai, et al. (2004). "Mycobacterium tuberculosis cell envelope lipids and the host immune response." Cell Microbiol 6(2): 105-116.

Karakousis, P. C., E. P. Williams, et al. (2008). "Altered expression of isoniazid-regulated genes in drug-treated dormant Mycobacterium tuberculosis." J Antimicrob Chemother 61(2): 323-331.

Klinkenberg, L. G., L. A. Sutherland, et al. (2008). "Metronidazole lacks activity against Mycobacterium tuberculosis in an in vivo hypoxic granuloma model of latency." Journal of Infectious Diseases 198(2): 275-283.

Kocagoz, T., C. J. Hackbarth, et al. (1996). "Gyrase mutations in laboratory-selected, fluoroquinolone-resistant mutants of Mycobacterium tuberculosis H37Ra." Antimicrob Agents Chemother 40(8): 1768-1774.

Kochi, A., B. Vareldzis, et al. (1993). "Multidrug-resistant tuberculosis and its control." Res Microbiol 144(2): 104-110.

Konno, K., F. M. Feldmann, et al. (1967). "Pyrazinamide susceptibility and amidase activity of tubercle bacilli." Am Rev Respir Dis 95(3): 461-469.

Koul, A., E. Arnoult, et al. (2011). "The challenge of new drug discovery for tuberculosis." Nature 469(7331): 483-490.

Lalloo, U. G. and A. Ambaram (2010). "New antituberculous drugs in development." Curr HIV/ AIDS Rep 7(3): 143-151.

Lee, A. S., A. S. Teo, et al. (2001). "Novel mutations in ndh in isoniazid-resistant Mycobacterium tuberculosis isolates." Antimicrob Agents Chemother 45(7): 21572159.

Lenaerts, A. J., V. Gruppo, et al. (2005). "Preclinical testing of the nitroimidazopyran PA-824 for activity against Mycobacterium tuberculosis in a series of in vitro and in vivo models." Antimicrob Agents Chemother 49(6): 2294-2301.

Leung, K. L., C. W. Yip, et al. (2010). "Usefulness of resistant gene markers for predicting treatment outcome on second-line anti-tuberculosis drugs." J Appl Microbiol 109(6): 2087-2094.

Mackaness, G. B. and N. Smith (1952). "The action of isoniazid (isonicotinic acid hydrazide) on intracellular tubercle bacilli." Am Rev Tuberc 66(2): 125-133.

Makarov, V., G. Manina, et al. (2009). "Benzothiazinones kill Mycobacterium tuberculosis by blocking arabinan synthesis." Science 324(5928): 801-804.

Manina, G., M. Bellinzoni, et al. (2010). "Biological and structural characterization of the Mycobacterium smegmatis nitroreductase $\mathrm{NfnB}$, and its role in benzothiazinone resistance." Mol Microbiol 77(5): 1172-1185. 
Marttila, H. J., H. Soini, et al. (1998). "A Ser315Thr substitution in KatG is predominant in genetically heterogeneous multidrug-resistant Mycobacterium tuberculosis isolates originating from the St. Petersburg area in Russia." Antimicrob Agents Chemother 42(9): 2443-2445.

Matsumoto, M., H. Hashizume, et al. (2006). "OPC-67683, a nitro-dihydro-imidazooxazole derivative with promising action against tuberculosis in vitro and in mice." PLoS Med 3(11): e466.

Matteelli, A., A. C. Carvalho, et al. (2010). "TMC207: the first compound of a new class of potent anti-tuberculosis drugs." Future Microbiol 5(6): 849-858.

Miesel, L., T. R. Weisbrod, et al. (1998). "NADH dehydrogenase defects confer isoniazid resistance and conditional lethality in Mycobacterium smegmatis." J Bacteriol 180(9): 2459-2467.

Mikusova, K., R. A. Slayden, et al. (1995). "Biogenesis of the mycobacterial cell wall and the site of action of ethambutol." Antimicrob Agents Chemother 39(11): 2484-2489.

Mitchison, D. A. (1985). "The action of antituberculosis drugs in short-course chemotherapy." Tubercle 66(3): 219-225.

Mitchison, D. A. (1985). "[Mechanisms of the action of drugs in the short-course chemotherapy]." Bull Int Union Tuberc 60(1-2): 36-40.

Morlock, G. P., B. Metchock, et al. (2003). "ethA, inhA, and katG loci of ethionamideresistant clinical Mycobacterium tuberculosis isolates." Antimicrob Agents Chemother 47(12): 3799-3805.

Musser, J. M. (1995). "Antimicrobial agent resistance in mycobacteria: molecular genetic insights." Clin Microbiol Rev 8(4): 496-514.

Musser, J. M., V. Kapur, et al. (1996). "Characterization of the catalase-peroxidase gene (katG) and inhA locus in isoniazid-resistant and -susceptible strains of Mycobacterium tuberculosis by automated DNA sequencing: restricted array of mutations associated with drug resistance." Journal of Infectious Diseases 173(1): 196-202.

Nair, J., D. A. Rouse, et al. (1993). "The rpsL gene and streptomycin resistance in single and multiple drug-resistant strains of Mycobacterium tuberculosis." Mol Microbiol 10(3): 521-527.

Ordway, D., M. Viveiros, et al. (2003). "Clinical concentrations of thioridazine kill intracellular multidrug-resistant Mycobacterium tuberculosis." Antimicrob Agents Chemother 47(3): 917-922.

Pasca, M. R., G. Degiacomi, et al. (2010). "Clinical isolates of Mycobacterium tuberculosis in four European hospitals are uniformly susceptible to benzothiazinones." Antimicrob Agents Chemother 54(4): 1616-1618.

Paulin, L. G., E. E. Brander, et al. (1985). "Specific inhibition of spermidine synthesis in Mycobacteria spp. by the dextro isomer of ethambutol." Antimicrob Agents Chemother 28(1): 157-159.

Petrella, S., E. Cambau, et al. (2006). "Genetic basis for natural and acquired resistance to the diarylquinoline R207910 in mycobacteria." Antimicrob Agents Chemother 50(8): 2853-2856.

Piscitelli, S. C., L. H. Danziger, et al. (1992). "Clarithromycin and azithromycin: new macrolide antibiotics." Clin Pharm 11(2): 137-152. 
Protopopova, M., C. Hanrahan, et al. (2005). "Identification of a new antitubercular drug candidate, SQ109, from a combinatorial library of 1,2-ethylenediamines." J Antimicrob Chemother 56(5): 968-974.

Quemard, A., J. C. Sacchettini, et al. (1995). "Enzymatic characterization of the target for isoniazid in Mycobacterium tuberculosis." Biochemistry 34(26): 8235-8241.

Ramaswamy, S. and J. M. Musser (1998). "Molecular genetic basis of antimicrobial agent resistance in Mycobacterium tuberculosis: 1998 update." Tuber Lung Dis 79(1): 329.

Ramaswamy, S. V., A. G. Amin, et al. (2000). "Molecular genetic analysis of nucleotide polymorphisms associated with ethambutol resistance in human isolates of Mycobacterium tuberculosis." Antimicrob Agents Chemother 44(2): 326-336.

Raynaud, C., M. A. Laneelle, et al. (1999). "Mechanisms of pyrazinamide resistance in mycobacteria: importance of lack of uptake in addition to lack of pyrazinamidase activity." Microbiology 145 ( Pt 6): 1359-1367.

Rengarajan, J., C. M. Sassetti, et al. (2004). "The folate pathway is a target for resistance to the drug para-aminosalicylic acid (PAS) in mycobacteria." Mol Microbiol 53(1): 275-282.

Robitzek, E. H. and I. J. Selikoff (1952). "Hydrazine derivatives of isonicotinic acid (rimifon marsilid) in the treatment of active progressive caseous-pneumonic tuberculosis; a preliminary report." Am Rev Tuberc 65(4): 402-428.

Rouse, D. A., J. A. DeVito, et al. (1996). "Site-directed mutagenesis of the katG gene of Mycobacterium tuberculosis: effects on catalase-peroxidase activities and isoniazid resistance." Mol Microbiol 22(3): 583-592.

Rozwarski, D. A., G. A. Grant, et al. (1998). "Modification of the NADH of the isoniazid target (InhA) from Mycobacterium tuberculosis." Science 279(5347): 98-102.

Saint-Joanis, B., H. Souchon, et al. (1999). "Use of site-directed mutagenesis to probe the structure, function and isoniazid activation of the catalase/peroxidase, KatG, from Mycobacterium tuberculosis." Biochem J 338 ( Pt 3): 753-760.

Sander, P., A. Meier, et al. (1996). "Ribosomal drug resistance in mycobacteria." Res Microbiol 147(1-2): 59-67.

Scorpio, A., P. Lindholm-Levy, et al. (1997). "Characterization of pncA mutations in pyrazinamide-resistant Mycobacterium tuberculosis." Antimicrob Agents Chemother 41(3): 540-543.

Scorpio, A. and Y. Zhang (1996). "Mutations in pncA, a gene encoding pyrazinamidase/nicotinamidase, cause resistance to the antituberculous drug pyrazinamide in tubercle bacillus." Nat Med 2(6): 662-667.

Sensi, P. (1983). "History of the development of rifampin." Rev Infect Dis 5 Suppl 3: S402406.

Sharma, S. K. and A. Mohan (2006). "Multidrug-resistant tuberculosis: a menace that threatens to destabilize tuberculosis control." Chest 130(1): 261-272.

Singh, R., U. Manjunatha, et al. (2008). "PA-824 kills nonreplicating Mycobacterium tuberculosis by intracellular NO release." Science 322(5906): 1392-1395.

Sreevatsan, S., X. Pan, et al. (1996). "Characterization of rpsL and rrs mutations in streptomycin-resistant Mycobacterium tuberculosis isolates from diverse geographic localities." Antimicrob Agents Chemother 40(4): 1024-1026. 
Sreevatsan, S., K. E. Stockbauer, et al. (1997). "Ethambutol resistance in Mycobacterium tuberculosis: critical role of embB mutations." Antimicrob Agents Chemother 41(8): 1677-1681.

Steele, M. A. and R. M. Des Prez (1988). "The role of pyrazinamide in tuberculosis chemotherapy." Chest 94(4): 845-850.

Stover, C. K., P. Warrener, et al. (2000). "A small-molecule nitroimidazopyran drug candidate for the treatment of tuberculosis." Nature 405(6789): 962-966.

Suter, E. (1952). "Multiplication of tubercle bacilli within phagocytes cultivated in vitro, and effect of streptomycin and isonicotinic acid hydrazide." Am Rev Tuberc 65(6): 775776.

Takayama, K., E. L. Armstrong, et al. (1979). "Inhibition by ethambutol of mycolic acid transfer into the cell wall of Mycobacterium smegmatis." Antimicrob Agents Chemother 16(2): 240-242.

Takayama, K., H. K. Schnoes, et al. (1975). "Site of inhibitory action of isoniazid in the synthesis of mycolic acids in Mycobacterium tuberculosis." J Lipid Res 16(4): 308317.

Takayama, K., L. Wang, et al. (1972). "Effect of isoniazid on the in vivo mycolic acid synthesis, cell growth, and viability of Mycobacterium tuberculosis." Antimicrob Agents Chemother 2(1): 29-35.

Taniguchi, H., B. Chang, et al. (1997). "Molecular analysis of kanamycin and viomycin resistance in Mycobacterium smegmatis by use of the conjugation system." Journal of Bacteriology 179(15): 4795-4801.

Telenti, A., P. Imboden, et al. (1993). "Detection of rifampicin-resistance mutations in Mycobacterium tuberculosis." Lancet 341(8846): 647-650.

Telenti, A., W. J. Philipp, et al. (1997). "The emb operon, a gene cluster of Mycobacterium tuberculosis involved in resistance to ethambutol." Nat Med 3(5): 567-570.

Tomasz, A., A. Albino, et al. (1970). "Multiple antibiotic resistance in a bacterium with suppressed autolytic system." Nature 227(5254): 138-140.

Trefzer, C., M. Rengifo-Gonzalez, et al. (2010). "Benzothiazinones: prodrugs that covalently modify the decaprenylphosphoryl-beta-D-ribose 2'-epimerase DprE1 of Mycobacterium tuberculosis." J Am Chem Soc 132(39): 13663-13665.

Tsukamura, M. and S. Mizuno (1975). "Cross-resistant relationships among the aminoglucoside antibiotics in Mycobacterium tuberculosis." J Gen Microbiol 88(2): 269-274.

van Soolingen, D., R. Hernandez-Pando, et al. (2010). "The antipsychotic thioridazine shows promising therapeutic activity in a mouse model of multidrug-resistant tuberculosis." Plos One 5(9).

Vannelli, T. A., A. Dykman, et al. (2002). "The antituberculosis drug ethionamide is activated by a flavoprotein monooxygenase." J Biol Chem 277(15): 12824-12829.

Via, L. E., P. L. Lin, et al. (2008). "Tuberculous granulomas are hypoxic in guinea pigs, rabbits, and nonhuman primates." Infection and Immunity 76(6): 2333-2340.

Vilcheze, C., Y. Av-Gay, et al. (2008). "Mycothiol biosynthesis is essential for ethionamide susceptibility in Mycobacterium tuberculosis." Mol Microbiol 69(5): 1316-1329.

Von Groll, A., A. Martin, et al. (2009). "Fluoroquinolone resistance in Mycobacterium tuberculosis and mutations in gyrA and gyrB." Antimicrob Agents Chemother 53(10): 4498-4500. 
Wade, M. M. and Y. Zhang (2004). "Mechanisms of drug resistance in Mycobacterium tuberculosis." Front Biosci 9: 975-994.

Wallis, R. S., W. Jakubiec, et al. (2011). "Biomarker-assisted dose selection for safety and efficacy in early development of PNU-100480 for tuberculosis." Antimicrob Agents Chemother 55(2): 567-574.

Wayne, L. G. and L. G. Hayes (1996). "An in vitro model for sequential study of shiftdown of Mycobacterium tuberculosis through two stages of nonreplicating persistence." Infection and Immunity 64(6): 2062-2069.

Wayne, L. G. and H. A. Sramek (1994). "Metronidazole is bactericidal to dormant cells of Mycobacterium tuberculosis." Antimicrob Agents Chemother 38(9): 2054-2058.

Weinstein, E. A., T. Yano, et al. (2005). "Inhibitors of type II NADH:menaquinone oxidoreductase represent a class of antitubercular drugs." Proc Natl Acad Sci U S A 102(12): 4548-4553.

Williams, K. N., S. J. Brickner, et al. (2009). "Addition of PNU-100480 to first-line drugs shortens the time needed to cure murine tuberculosis." Am J Respir Crit Care Med 180(4): 371-376.

Winder, F. G. and P. B. Collins (1970). "Inhibition by isoniazid of synthesis of mycolic acids in Mycobacterium tuberculosis." J Gen Microbiol 63(1): 41-48.

Wong, S. Y., J. S. Lee, et al. (2011). "Mutations in gidB confer low-level streptomycin resistance in Mycobacterium tuberculosis." Antimicrob Agents Chemother 55(6): 2515-2522.

Xu, C., B. N. Kreiswirth, et al. (1996). "Fluoroquinolone resistance associated with specific gyrase mutations in clinical isolates of multidrug-resistant Mycobacterium tuberculosis." Journal of Infectious Diseases 174(5): 1127-1130.

Yeager, R. L., W. G. Munroe, et al. (1952). "Pyrazinamide (aldinamide) in the treatment of pulmonary tuberculosis." Am Rev Tuberc 65(5): 523-546.

Zatman, L. J., N. O. Kaplan, et al. (1954). "Effect of isonicotinic acid hydrazide on diphosphopyridine nucleotidases." J Biol Chem 209(2): 453-466.

Zhang, N., J. B. Torrelles, et al. (2003). "The Emb proteins of mycobacteria direct arabinosylation of lipoarabinomannan and arabinogalactan via an N-terminal recognition region and a C-terminal synthetic region." Mol Microbiol 50(1): 69-76.

Zhang, T., S. Y. Li, et al. (2011). "Short-course Chemotherapy with TMC-207 and Rifapentine in a Murine Model of Latent Tuberculosis Infection." Am J Respir Crit Care Med.

Zhang, Y. (2005). "The magic bullets and tuberculosis drug targets." Annu Rev Pharmacol Toxicol 45: 529-564.

Zhang, Y., B. Heym, et al. (1992). "The catalase-peroxidase gene and isoniazid resistance of Mycobacterium tuberculosis." Nature 358(6387): 591-593.

Zhang, Y., A. Scorpio, et al. (1999). "Role of acid pH and deficient efflux of pyrazinoic acid in unique susceptibility of Mycobacterium tuberculosis to pyrazinamide." Journal of Bacteriology 181(7): 2044-2049.

Zhang, Y., M. M. Wade, et al. (2003). "Mode of action of pyrazinamide: disruption of Mycobacterium tuberculosis membrane transport and energetics by pyrazinoic acid." J Antimicrob Chemother 52(5): 790-795. 


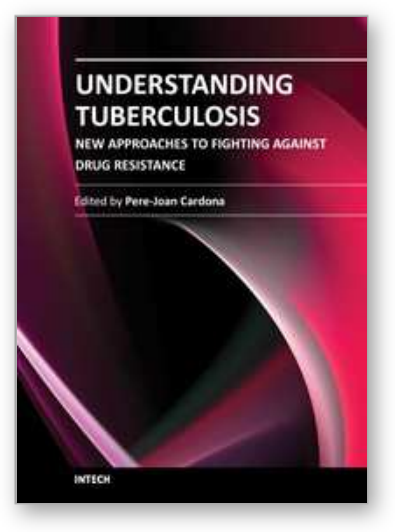

\author{
Understanding Tuberculosis - New Approaches to Fighting \\ Against Drug Resistance \\ Edited by Dr. Pere-Joan Cardona
}

ISBN 978-953-307-948-6

Hard cover, 376 pages

Publisher InTech

Published online 15, February, 2012

Published in print edition February, 2012

In 1957, a Streptomyces strain, the ME/83 (S.mediterranei), was isolated in the Lepetit Research Laboratories from a soil sample collected at a pine arboretum near Saint Raphael, France. This drug was the base for the chemotherapy with Streptomicine. The euphoria generated by the success of this regimen lead to the idea that TB eradication would be possible by the year 2000. Thus, any further drug development against TB was stopped. Unfortunately, the lack of an accurate administration of these drugs originated the irruption of the drug resistance in Mycobacterium tuberculosis. Once the global emergency was declared in 1993, seeking out new drugs became urgent. In this book, diverse authors focus on the development and the activity of the new drug families.

\title{
How to reference
}

In order to correctly reference this scholarly work, feel free to copy and paste the following:

Anastasia S. Kolyva and Petros C. Karakousis (2012). Old and New TB Drugs: Mechanisms of Action and Resistance, Understanding Tuberculosis - New Approaches to Fighting Against Drug Resistance, Dr. PereJoan Cardona (Ed.), ISBN: 978-953-307-948-6, InTech, Available from:

http://www.intechopen.com/books/understanding-tuberculosis-new-approaches-to-fighting-against-drugresistance/old-and-new-tb-drugs-mechanisms-of-action-and-resistance

\section{INTECH}

open science | open minds

\author{
InTech Europe \\ University Campus STeP Ri \\ Slavka Krautzeka 83/A \\ 51000 Rijeka, Croatia \\ Phone: +385 (51) 770447 \\ Fax: +385 (51) 686166 \\ www.intechopen.com
}

\author{
InTech China \\ Unit 405, Office Block, Hotel Equatorial Shanghai \\ No.65, Yan An Road (West), Shanghai, 200040, China \\ 中国上海市延安西路65号上海国际贵都大饭店办公楼 405 单元 \\ Phone: +86-21-62489820 \\ Fax: +86-21-62489821
}


(C) 2012 The Author(s). Licensee IntechOpen. This is an open access article distributed under the terms of the Creative Commons Attribution 3.0 License, which permits unrestricted use, distribution, and reproduction in any medium, provided the original work is properly cited. 